\title{
OCORRÊNCIA DE DERMATÓFITOS EM TEGUMENTO DE BOVINOS E OVINOS HÍGIDOS
}

\author{
Flavio Oliveira Surpilli ${ }^{1}$ \\ Igor Renan Honorato Gatto ${ }^{2}$ \\ Danila Fernanda Rodrigues Frias ${ }^{1}$ \\ Dora Inés Kozusny-Andreani ${ }^{1}$
}

SURPILLI, F. O.; GATTO, I. R. H.; FRIAS, D. F. R.; KOZUSNY-ANDREANI, D. I. Ocorrência de dermatófitos em tegumento de bovinos e ovinos hígidos. Arq. Ciênc. Vet. Zool. UNIPAR, Umuarama, v. 21, n. 1, p. 9-12, jan./mar. 2018.

\begin{abstract}
RESUMO: O objetivo desta pesquisa foi verificar a prevalência de dermatófitos em tegumento de bovinos e ovinos hígidos e a sua capacidade de transmissão e desenvolvimento da doença. Para isso, foram coletadas amostras de pelo e descamações de 90 bovinos e 90 ovinos hígidos. Essas amostras foram semeadas em meio DTM e ágar Sabouraud Dextrose enriquecido com extrato de levedura, tiamina, antibióticos (estreptomicina e cloranfenicol) e suplementado de cicloheximida, sendo essas incubadas a $35{ }^{\circ} \mathrm{C}$ por 10 dias. As culturas positivas foram avaliadas macro e microscopicamente e, os fungos foram identificados por métodos bioquímicos. Verificou-se que as espécies isoladas com maior frequência, nos bovinos, foram $T$. mentagrophytes e M. gypseum. Nos ovinos, foi constatada maior ocorrência do agente T. verrucosum, seguido por T. mentagrophytes e M. gypseum. Houve também isolamento de M. canis, porém, em um número reduzido de amostras. Assim, concluiu-se que o tegumento de bovinos e de ovinos hígidos apresentou incidência elevada de dermatófitos de diferentes espécies, em amostras coletadas durante o período chuvoso do ano. Por isso, em animais jovens ou naqueles submetidos a elevados níveis de estresse e, consequente queda da resposta imunológica, o risco de desenvolvimento da dermatofitose e da transmissão dos dermatófitos neste período são eminentes.
\end{abstract}

PALAVRAS-CHAVE: Dermatofitose. Microsporum. Pelos. Trichophyton.

\section{OCCURRENCE OF DERMATOPHYTES IN HEALTHY CATTLE AND SHEEP TEGUMENT}

\begin{abstract}
The purpose of this study was to determine the prevalence of dermatophytes in healthy cattle and sheep teguments, and their ability to transmit and develop diseases. Samples of fur and flaking from 90 healthy cattle and 90 healthy sheep were collected. These samples were plated on DTM medium and Sabouraud Dextrose agar supplemented with yeast extract, thiamine, antibiotics (streptomycin and chloramphenicol) enhanced with cycloheximide. They were then incubated at $35{ }^{\circ} \mathrm{C}$ for 10 days. Positive cultures were macroscopically and microscopically evaluated, and fungi were identified by biochemical methods. It was found that the most frequent species isolated in cattle were T. mentagrophytes and M. gypseum. In sheep, a higher occurrence of the agent T. verrucosum could be observed, followed by T. mentagrophytes and M. gypseum. There was also the isolation of $M$. canis, but in a small number of samples. Thus, it could be concluded that the tegument of healthy cattle and sheep showed high incidence of dermatophytes from different species in samples collected during the rainy season of the year. Therefore, in young animals, in those subjected to high levels of stress and consequent drop in immune response, there is eminent risk of developing dermatophytosis and transmission of dermatophytes in this period.
\end{abstract}

KEYWORDS: Dermatophytosis. Fur. Microsporum. Trichophyton.

\section{OCURRENCIA DE DERMATOFITOS EN TEGUMENTO DE BOVINOS Y OVINOS HÍGIDOS}

RESUMEN: El objetivo de esta investigación ha sido verificar la prevalencia de dermatofitos en tegumento de bovinos y ovinos hígidos y su capacidad de transmisión y desarrollo de la enfermedad. Para ello, se recolectó muestras de pelo y descamaciones de 90 bovinos y 90 ovinos hígidos. Esas muestras fueron sembradas en medio DTM y agar Sabouraud Dextrosis enriquecida con extracto de levadura, tiamina, antibióticos (estreptomicina y cloranfenicol) y suplementado de cicloheximida, siendo esas incubadas a $35^{\circ} \mathrm{C}$ durante 10 días. Los cultivos positivos fueron evaluados macro y microscópicamente y los hongos fueron identificados por métodos bioquímicos. Se verificó que las especies aisladas con mayor frecuencia, en los bovinos, fueron T. mentagrophytes y $M$. gypseum. En los ovinos, se constató mayor ocurrencia del agente T. verrucosum, seguido por T. mentagrophytes y M. gypseum. También hubo aislamiento de $M$. canis, sin embargo, en un número reducido de muestras. Así, se concluyó que el tegumento de bovinos y de ovinos hígidos presentó una elevada incidencia de dermatofitos de diferentes especies, en muestras recogidas durante el período lluvioso del año. Por lo tanto, en animales jóvenes o en aquellos sometidos a altos niveles de estrés y, consecuente caída de la respuesta inmunológica, el riesgo de desarrollo de la dermatofitosis y de la transmisión de los dermatofitos en este período son eminentes.

PALABRAS CLAVE: Dermatofitosis. Microsporum. Pelo. Trichophyton.

DOI: 10.25110 /arqvet.v21i1.2018.5678

${ }^{1}$ Universidade Brasil, Campus de Fernandópolis, SP. danila.frias@universidadebrasil.edu.br

${ }^{2}$ Universidade Estadual Paulista - UNESP, Jaboticabal, SP. 


\section{Introdução}

Os dermatófitos constituem um grupo de fungos que apresentam capacidade de invadir tecidos queratinizados do homem e de animais, causando dermatofitose, doença de carácter contagioso e de elevada prevalência mundial, sendo considerada uma das zoonoses mais comuns em todo o mundo (FARIAS et al., 2011).

Os dermatófitos são divididos em grupos, de acordo com o seu habitat natural, sendo classificados como geofílicos, zoofílicos e antropofílicos (CABAÑES, 2000; MENELAOS, 2006; QUINN et al., 2011). Os fungos zoofílicos são isolados de animais, e possuem a capacidade de causar doenças em seres humanos; os geofílicos são encontrados no solo e ocasionalmente infectam o homem, enquanto que os antropofílicos são restritos a seres humanos e raramente infectam animais (HIRSH; ZEE, 2003; QUINN et al., 2011).

No grupo dos zoofílicos, destacam-se três gêneros: Microsporum, Trichophyton e Epidermophyton (QUINN et al., 2011; TORTORA et al., 2012). Os gêneros Microsporum e Trichophyton estão associados, frequentemente, com dermatofitoses em animais domésticos, tendo como reservatório principal os bovinos, gatos e cães (CABAÑES, 2000; PAPINI et al., 2009; GANGIL et al., 2012). Trichophyton verrucosum é considerado um dos dermatófitos mais comuns em bovinos, caprinos e ovinos, e a dermatofitose bovina é uma zoonose que pode causar perdas significativas (SILVERet al., 2008).

Segundo Nweze (2010), embora a dermatofitose apresente baixas taxas de mortalidade, a afecção pode ser epizoótica e pode gerar prejuízos econômicos significativos, resultante da perda de produção, principalmente em casos de doença generalizada, devido à diminuição de peso corporal, ao abate precoce e a classificação inferior de couros e peles dos animais acometidos. Além disto, segundo o mesmo autor, o tratamento é de alto custo e a doença é considerada um grave problema de saúde pública por ser uma zoonose. Por este motivo, o objetivo desta pesquisa foi verificar a prevalência destes dermatófitos em tegumento de bovinos e ovinos hígidos, a capacidade de sua transmissão e de desenvolvimento da doença.

\section{Materiais e Métodos}

Foram coletadas amostras de pelos e descamações de 90 bovinos e 90 ovinos oriundos de clínicas veterinárias e do Hospital Veterinário da Universidade Brasil, Região de Fernandópolis, SP.
Antes do início da pesquisa o projeto foi aprovado pela CEUA da Unicastelo (protocolo 1-00001/2012).

O material foi colhido de forma asséptica, empregando a técnica descrita por Mariat e Adan-Campos (1967). As amostras foram colhidas com utilização de pinça esterilizada na região da "tábua do pescoço", e os pelos e as escamas foram depositados em frascos esterilizados. Os pelos e as descamações cutâneas foram examinados para a detecção de elementos fúngicos (hifas e artrósporos) por meio de exame microscópico direto com hidróxido de potássio a $20 \%$ após leve aquecimento.

Para o isolamento dos dermatófitos, as amostras foram inoculadas em meio de DTM (Oxoid®) e ágar Sabouraud Dextrose (Oxoid $\AA$ ) enriquecido com extrato de levedura, tiamina, antibióticos (estreptomicina e cloranfenicol) e cicloheximida, incubadas a $35^{\circ} \mathrm{C}$ por 10 dias, sendo avaliadas a partir do terceiro dia de incubação para verificação do estabelecimento da cultura, no entanto o diagnóstico foi realizado no décimo dia.

A identificação dos dermatófitos baseou-se no exame macroscópico das colônias e pelo exame microscópico com o lactofenolazul de algodão. Foram consideradas positivas apenas aquelas amostras que em cultura desenvolveram dermatófitos, independentemente dos resultados obtidos no exame microscópico direto.

O exame microscópico foi realizado utilizando a técnica descrita por Minami (2003). Adicionou-se em uma lâmina uma gota de lactofenol azul-algodão. Em seguida, foi retirado um fragmento das bordas da cultura e depositado sobre a gota de corante e, posteriormente, foi depositada a lamínula. Após este procedimento, as estruturas foram observadas ao microscópio óptico (aumento de 400x). Para identificação macroscópica, foram avaliadas as características das colônias, levando-se em consideração o anverso e reverso das mesmas, como descrito por De laMaza et al. (1999) e Minami (2003). Sempre que necessário, realizou-se testes complementares, como a prova da uréase, para identificação.

Os dados obtidos foram tabulados e analisados pelo teste $\mathrm{F}$ de análise de variância e as médias comparadas pelo teste de Tukey ao nível de 5\% de probabilidade (SILVA; AZEVEDO, 2009).

\section{Resultados}

Das amostras de pelos e escamas de pele colhidas de bovinos $(\mathrm{n}=90)$ e ovinos $(\mathrm{n}=90)$, foram isolados dermatófitos das espécies Microsporum canis, M. gypseum, Trichophyton verrucosum e T. mentagrophytes (Tabela 1).

Tabela 1: Isolamento de dermatófitos em tegumento de bovinos e ovinos hígidos.

\begin{tabular}{l|c|c|c|c}
\hline \multicolumn{1}{c|}{ Dermatófitos } & Bovinos & & Ovinos & \\
\cline { 2 - 5 } & Número & \% & Número & \% \\
\hline Microsporum canis & $12 \mathrm{~d}$ & 13,33 & $6 \mathrm{c}$ & 6,67 \\
\hline Microsporum gypseum & $21 \mathrm{~b}$ & 23,33 & $24 \mathrm{~b}$ & 26,66 \\
\hline Trichophyton mentagrophytes & $18 \mathrm{c}$ & 20,00 & $24 \mathrm{~b}$ & 26,66 \\
\hline Trichophyton verrucossum & $9 \mathrm{e}$ & 10,00 & $30 \mathrm{a}$ & 33,34 \\
\hline Negativo & $30 \mathrm{a}$ & 33,34 & $6 \mathrm{c}$ & 6,67 \\
\hline Valor p & $* *$ & $* *$ & $* *$ & $* *$ \\
\hline
\end{tabular}

*Letras iguais na mesma coluna não diferem estatisticamente pelo teste de Duncan ao nível de 5\% de probabilidade.

**Valores de $\mathrm{p}<0,05$ apresentam significância estatística. 
As espécies isoladas com maior frequência entre os bovinos foram $T$. mentagrophytes e $M$. gypseum, enquanto que $M$. canise e $T$. verrucosum foram isolados em um número menor de amostras analisadas. Em 30 amostras, não houve isolamento de nenhum gênero de dermatófitos.

Nos ovinos, T. verrucosum apresentou maior prevalência, seguido de $T$. mentagrophytes e $M$. gypseum. A presença destas espécies de fungos foi significativamente superior aos isolados de M. canis. Apenas seis amostras foram negativas.

Em ovinos, o Trichophyton verrucosum e T. mentagrophytes foram os mais isolados, enquanto o Microsporum canis foi isolado em maior proporção em bovinos (Tabela 2).

Tabela 2: Proporção de dermatófitos isolados de tegumento de bovinos e ovinos.

\begin{tabular}{|c|c|c|c|}
\hline Dermatófitos & Bovinos & Ovinos & Valor $\mathbf{p}$ \\
\hline Microsporum canis & $12 \mathrm{a}$ & $6 \mathrm{~b}$ & $* *$ \\
\hline Microsporum gypseum & $21 \mathrm{a}$ & $24 \mathrm{a}$ & NS \\
\hline Trichophyton mentagrophytes & $18 \mathrm{~b}$ & $24 \mathrm{a}$ & $* *$ \\
\hline Trichophyton verrucossum & $9 \mathrm{~b}$ & $30 \mathrm{a}$ & $* *$ \\
\hline Negativo & $30 \mathrm{a}$ & $6 \mathrm{~b}$ & $* *$ \\
\hline
\end{tabular}

*Letras iguais na mesma linha não diferem estatisticamente pelo teste de Duncan a 5\% de probabilidade.

**Valores de $\mathrm{p}<0,05$ apresentam significância estatística.

NS: não significativo

\section{Discussão}

Os dermatófitos mais isolados no presente estudo foram das espécies Microsporum canis, M. gypseum, Trichophyton verrucosum e T. mentagrophytes. Resultados semelhantes foram obtidos por Amaral et al. (2011), que analisaram 672 amostras de pelos e escamas de pele obtidas de bovinos de corte da fronteira Oeste do Rio Grande do Sul, verificando a ocorrência dos dermatófitos M.gypseum, T. mentagrophytes e T. veruucosum. Estes autores destacaram que o isolamento destes dermatófitos ocorreu durante os meses de inverno, e, por isto, poucas amostras foram positivas (apenas seis). No presente trabalho, as amostragens foram realizadas no período de novembro a março (2014-2015). Nesta época do ano, na região Noroeste Paulista, há grande incidência de chuvas. Por isso, acredita-se que o alto índice de isolamento destes dermatófitos esteja relacionado à alta umidade relativa do ar e à menor incidência de luz solar, que favoreceriam o desenvolvimento dos mesmos (SINGH et al., 1997).

Registros sobre dermatófitos em tegumento de ovinos são escassos. Cabañes (2000) relata que T. verrucosum é o agente de dermatofitose citado com maior frequência, e que $T$. mentagrophytes, $M$. canis e $M$. gypseum também podem ser isolados de lesões dermatofíticas em ruminantes, porém, não existem relatos em ovinos hígidos.

Em pesquisa realizada no Rio Grande do Sul, a frequência de $T$. verrucosum em bovinos hígidos foi de 1,2\%, demostrando que, embora este dermatófito seja zoofílico e constituinte da microbiota de bovinos hígidos, é isolado em baixas proporções (CABAÑES, 2000; SILVA; AZEVEDO, 2009; AMARAL et al., 2011). No presente trabalho, T. verrucosum foi isolado em $10 \%$ das amostras, corroborando com os resultados obtidos por Singh et al. (1997) e por Moretti et al. (1998).

Notou-se que a incidência de dermatófitos em pelos e escamas cutâneas de ovinos foi superior quando comparada as amostras provenientes de bovinos. Acredita-se que este fato esteja relacionado às características da pele dos ovinos, pois estes possuem pelos maiores e mais densos que os bovinos, mantendo, desta forma, um ambiente de umidade e temperatura favorável ao desenvolvimento de dermatófitos.
Os dermatófitos isolados no presente estudo são os principais agentes causadores da dermatofitose em bovinos e ovinos, principalmente Trichophyton verrucosum (CABAÑES, 2000; SILVEIRA et al., 2003; YILDIRIM et al., 2010; AMARAL et al., 2011; DUARTE et al., 2013).

Alta incidência de $T$. verrucosum em bovinos com lesões cutâneas foi verificada no Japão $(58,6 \%)$, na Tanzânia $(33,3 \%)$, na Turquia (38\%) e no Brasil (33\% a 95,8\%) (TAKATORI et al., 1990; OLHOFF, 2003; SILVEIRA et al., 2003; AMARAL et al., 2011; SWAI; SANKA, 2012).

A presença de $T$. verrucosum em bovinos hígidos é controversa. Segundo Silveira et al. (2003), este dermatófito é um fungo de transição, ocasional e não residente da pele de bovinos. Já Amaral et al. (2011) afirmam que bovinos sadios não atuam como portadores assintomáticos de $T$. verrucosum e que esta espécie de dermatófito não é residente no tegumento dos animais, sendo a sua prevalência influenciada pela estação do ano.

Londero et al. (1970) afirmaram que os bovinos são responsáveis pela perpetuação da doença, atuando como disseminadores do agente. Neste trabalho, o mesmo foi isolado em $10 \%$ das amostras coletadas de bovinos hígidos e em $33 \%$ das amostras de ovinos hígidos. Acredita-se que tal fato se deu por conta da estação do ano chuvosa em que foram realizadas as coletas, assim como os dados encontrados por Amaral et al., (2011).

\section{Conclusão}

De acordo com a metodologia utilizada e os resultados obtidos, concluiu-se que o tegumento de bovinos e de ovinos hígidos apresentou incidência elevada de dermatófitos de diferentes espécies, em amostras coletadas durante o período chuvoso do ano. Por isso, em animais jovens ou com elevado nível de estresse, levando à queda de imunidade, o risco de desenvolvimento da dermatofitose e transmissão dos dermatófitos neste período são eminentes.

\section{Referências}

AMARAL, C. D. P.; PEREIRA, D. I. B.; MEIRELES, M. C. A. Caracterização da microbiota no tegumento hígido de 
bovinos de corte por fungos filamentosos. Ciência Rural, v. 41, n. 12, p. 2137-2142, 2011.

CABAÑES, F. J. Dermatophytes in domestics animais. Revista Iberoamericana de Micología, v. 17, p. 104-108, 2000 .

DE LA MAZA, L. M.; PEZZLO, M. T.; BARON, E. J. Atlas de diagnóstico em microbiologia. Porto Alegre: Artmed, 1999. 216 p.

DUARTE, E. R.; OLIVEIRA, N. J. F.; ROSA, C. A.; FACURY-FILHO, E. J. Yeast isolated from beef heifers with ringworm. Archivos de Medicina Veterinária, v. 45, p. 71-75, 2013.

FARIAS, M. R.; CONDAS, L. A. Z.; RAMALHO, F.; BIER, D.; MURO, M. D.; PIMPÃO, C. T. Avaliação do estado de carreador assintomático de fungos dermatofitos em felinos (Feliscatus - Linnaeus, 1793) destinados à doação em centros de controle de zoonoses e sociedades protetoras de animais. Veterinária e Zootecnia, v. 18, n. 2, p. 306-312, 2011.

GANGIL, R.; DUTTA, P.; TRIPATHI, R.; SINGATHIA, R.; LAKHOTIA, R. L. Incidence of dermatophytosis in canine cases presented at Apollo Veterinary College, Rajashtan, India. Veterinary World, v. 5, n. 11, p. 682-684, 2012.

HIRSH, D. C.; ZEE, Y. C. MicrobiologiaVeterinária. Rio de Janeiro: Guanabara Koogan, 2003. 446 p.

LONDERO, A.T.; FISCHMAN, O.; LOPES, J. O. Isolamento do Trichophyton mentagrophytes de bovinos clinicamente sãos. Revista Brasileira de Pesquisas Médicas e Biológicas, v.3, n.3-4, p.27-28, 1970.

MARIAT, F.; ADAN-CAMPOS, C. La techinique du carré du tapis, méthode simple de prévelement dans les mycoses superficialles. Annales de l'Institut Pasteur, v. 113, p. 666-668, 1967.

MENELAOS, L. A. Dermatophytes in dog and cat. Buletin of University of Agricultural Sciences and Veterinary Medicine Cluj-Napoca, v. 23, p. 304-308, 2006.

MINAMI, P. S. Micologia. Métodos laboratoriais de diagnósticos das micoses. Barueri: Manole, 2003. 199 p.

MORETTI, A.; BONCIO, L.; PASQUALI, P.; PIERGILI FIORETTI, D. Epidemiological aspects of dermatophyte infections in horses and catlle. Zentralbl Veterinarmed B, v.45, n.4, p.205-208, 1998.

NWEZE, E. I. Dermatophytosis among children of Fulani/ Hausaherdsmen living in islated camps south eastern Nigeria. Revista Iberoamericana de Micología, v. 27, n. 4, p. 191-194, 2010.

OLHOFF, R. D. Exame micológico e acompanhamento clínico de bovinos infectados pela forma latente de Trichophyton verrucosum (Bodin, 1902). Archives of
Veterinary Science, v. 8, n. 2, p. 47-50, 2003.

PAPINI, R.; NARDONI, S.; FANELLI, A.; MANCIANTI, F. High infection rate Trichophyton verrucosum in calves from central Italy. Zoonoses Public Health, v. 56, n. 2, p. 59-64, 2009.

QUINN, P. J.; MARKEY, B. K.; LEONARD, F. C.; FITZPATRICK, E. S.; FANNING, S.; HARTIGAN, P. J. Veterinary microbiology and microbial disease. 2 ed. Oxford: Blackwell Science Ltda, 2011. 512 p.

SILVA, F. A. S. E.; AZEVEDO, C. A. V. Principal Components Analysis in the Software Assistat-Statistical Attendance. In: WORLD CONGRESS ON COMPUTERS IN AGRICUlTURE, 7, 2009, Reno. Proceedings.

Michigan: American Society of Agricultural and Biological Engineers, 2009.

SILVEIRA, E. S.; NOBRE, M. O.; SOUZA, L. L.; FARIA, R. O.; CLEFF, M. B.; MEIRELES, M. C. A. Trichophyton verrucosum em bovinos com pele hígida e com lesões. Acta Scientiae Veterinariaie, v. 31, n. 1, p. 45-49, 2003.

SILVER, S.; VINH, D. C.; EMBIL, J. M. The man who got too close to his cows. Diagnostic Microbiology and Infectious Disease, v. 60, n. 4, p. 419-420, 2008.

SINGH, A. P.; YADAV, T. L. J. S.; SINGH, A. P.; SHARMA, S. N. Clinico-epidedemiological studies on bovine dermatophytoses in and around Bikaner. Indian Journal of Animal Science, v. 68, p. 845-848, 1997.

SWAI, E. S.; SANKA, P. N. Bovine dermatophytosis caused by Trichophyton verrucosum: a case report. Veterinary World, v. 5, n. 5, p. 297-300, 2012.

TAKATORI, K.; KAWAI, S.; TAKAHASHI, A. Isolation of Trichophyton verrucosum from soil in cattle breeding environment. Japanese Journal of Veterinary Science, v. 52 , p. $823-825,1990$.

TORTORA, G. J.; FUNKE, B. R.; CASE, C. L. Microbiologia. Porto Alegre: Artmed, 2012. 934 p.

YILDIRIM, M.; CINAR, M.; OCAL, N.; YAGCI, B. B.; ASKAR, S. Prevalence of clinical dermatophytes and oxidative stress in cattle. Journal of Animal and Veterinary Advances, v. 9, n. 14, p. 1978-1982, 2010 .

Recebido em: 08.04.2016 Aceito em: 27.04.2018 